\title{
Boron nitride nanotube-based amphiphilic hybrid nanomaterials for superior encapsulation of hydrophobic cargos
}

\section{Abu Saleh, Doaa}

2017-12

Abu Saleh, D , Niskanen , J , Xue , Y, Golberg , D , Winnik , F M \& Sosnik , A 2017 , '

Boron nitride nanotube-based amphiphilic hybrid nanomaterials for superior encapsulation of

hydrophobic cargos ' , Materials today chemistry , vol. 6 , pp. 45-50 . https://doi.org/10.1016/j.mtchem.2017.09.003

http://hdl.handle.net/10138/307994

https://doi.org/10.1016/j.mtchem.2017.09.003

cc_by_nc_nd

acceptedVersion

Downloaded from Helda, University of Helsinki institutional repository.

This is an electronic reprint of the original article.

This reprint may differ from the original in pagination and typographic detail.

Please cite the original version. 


\section{Boron Nitride Nanotube-Based Amphiphilic Hybrid Nanomaterials for Superior Encapsulation of Hydrophobic Cargos}

Doaa Abu Saleh, ${ }^{1}$ Jukka Niskanen, ${ }^{2}$ Yanming Xue, ${ }^{3}$ Dmitri Golberg ${ }^{3,4}$, Françoise M Winnik $^{2,5}$ and Alejandro Sosnik ${ }^{1, *}$

${ }^{1}$ Laboratory of Pharmaceutical Nanomaterials Science, Department of Materials Science and Engineering, Technion-Israel Institute of Technology, Haifa, Israel

${ }^{2}$ Laboratory of Polymer Chemistry, Department of Chemistry, A.I. Virtasen aukio 1, P.O. Box 55, 00014 University of Helsinki, Helsinki, Finland

${ }^{3}$ International Center for Materials Nanoarchitectonics (MANA), National Institute for Materials Science (NIMS), Tsukuba, Japan

${ }^{4}$ Queensland University of Technology, Brisbane, Qld, Australia

${ }^{5}$ Département de Chimie, Université de Montréal, CP 6128 Succursale Centre-Ville, Montréal, QC, Canada

*Corresponding Author:

Prof. Alejandro Sosnik

Department of Materials Science and Engineering

De-Jur Building, Office 607

Technion-Israel Institute of Technology

Technion City

3200003 Haifa, Israel

Phone \#: +972-077-887-1971

E-mail: alesosnik@gmail.com, sosnik@technion.ac.il

Keywords: Amphiphilic hybrid nanomaterials; boron nitride nanotubes; polymeric micelles; drug encapsulation; curcumin 
Abstract. We report an organic-inorganic hybrid core-shell nanomaterial obtained by conjugation of an amphiphilic monomethoxy-poly(ethylene glycol)- $b$-poly(epsiloncaprolactone) diblock copolymer to hydroxylated boron nitride nanotubes (BNNTs). The extent of copolymer grafting reached $64 \% \mathrm{w} / \mathrm{w}$, an exceptionally high value. The hybrid materials exhibit excellent physical stability in water and an outstanding loading capacity $(31.3 \% \mathrm{w} / \mathrm{w})$ for curcumin, a hydrophobic drug. Moroever, they present good compatibility with the Caco2 cell line, a model of intestinal epithelium. Our findings demonstrate the potential of multifunctional hybrid BNNTs to serve as a platform for complex amphiphilic nanoparticle architectures with improved features.

\section{Graphical abstract}

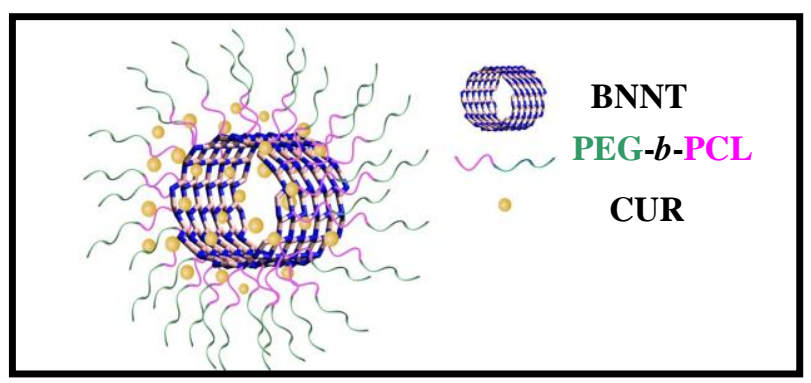


Core-shell nanostructures, have been investigated extensively for the encapsulation, delivery, and targeting of hydrophobic drugs. In this context, polymeric micelles have reached clinical trials in cancer therapy. ${ }^{1-4}$ However, a main disadvantage is their limited physical stability under extreme dilution in body fluids. ${ }^{5}$ Our recent efforts are focused on innovative approaches towards the design and synthesis of amphiphilic nanomaterials that, on the one hand, are as effective as polymeric micelles in terms of hydrophobic drug encapsulation, and, on the other, display maximum physical stability and resistance against dilution. In the past, we employed nanodiamonds as particulate anchors for the conjugation of polymeric amphiphiles made of poly(ethylene glycol)$b$-poly(epsilon-caprolactone) (PEG- $b$-PCL). ${ }^{6}$ These core-anchored nanomaterials were very stable in suspension, encapsulated up to $17.5 \% \mathrm{w} / \mathrm{w}$ of the hydrophobic drug nitazoxanide and sustained its release in vitro by anomalous transport. This was a significant finding, since most strategies proposed to stabilize polymeric micelles tend to decrease their loading capacity and/or compromise the integrity and the activity of the encpasulated cargo. ${ }^{5,7} \mathrm{We}$ are interested to explore the versatility of this approach and to extend it to inorganic nanomaterials with different nanostructures and morphoplogies.

Boron nitride nanotubes (BNNTs), exceptionally strong and inert materials, ${ }^{8}$ were shown to be non-toxic as long as their length does not exceed a few microns. ${ }^{9}$ Pristine BNNTs are hydrophobic and do not disperse well in water, a fundamental drawback towards their utilization in biomedical applications. Their dispersibility in aqueous media is improved upon adsorption of water-soluble polymers, ${ }^{10,11}$ such as poly(Llysine $)^{12}$, or by exposing BNNTs to ammonia plasma, which increases the number of active $\mathrm{N}$ sites. Ammonia plasma is believed to generate a coordination bond through charge transfer from $\mathrm{N}$ to $\mathrm{B}$, when the energy barrier is below $0.45 \mathrm{eV} .^{13,14}$ A milder approach consists in treating BNNT dispersions in hydrophilic solvents with hydrophilic primary amines, such as glycine or aminoethanol that form a strong $\mathrm{N}-\mathrm{B}$ charge transfer bond interaction. ${ }^{15,16}$ It is also possible to oxidize B with concentrated nitric acid to produce $-\mathrm{OH}$ moieties. ${ }^{17}$ These strategies paved the way to improve the physical stability of BNNT aqueous dispersions. ${ }^{13,17}$ Following a similar concept, Weng et al. reported on the synthesis of hydrophilic BNNTs by the thermal substitution of $\mathrm{C}$ atoms in graphitic carbon nitrides with boric acid, forming $\mathrm{BN}(\mathrm{OH})$ with high hydroxylation degree. ${ }^{18}$ These materials can incorporate large payloads $(\sim 300 \mathrm{wt} \%)$ of 
the water-soluble anti-cancer drug doxorubicin for stronger activity in vitro. However, these chemical modifications did not improve the encapsulation of hydrophobic molecules, which represent $>70 \%$ of the drugs on the market and the new chemical entities under investigation. Recently, Niskanen et al. demonstrated that the inner channel of glycine-coated BNNTs can be loaded efficiently with hydrophobic drugs and serve as intracellular nano-delivery agents. ${ }^{16}$ To the best of our knowledge, this represents the first report ever on the encapsulation of a hydrophobic cargo within BNNTs. Rod-shaped nanoparticles are known to be internalized efficiently by cells via active uptake. ${ }^{19,20}$ These properties make BNNTs appealing candidates for encapsulation and delivery applications.

BNNTs display extremely high surface-to-mass ratio ${ }^{21}$ which, a priori, enables higher weight ratio of surface modification than other very dense ceramic nanomaterials currently under investigation (e.g., nanodiamonds). We hypothesized that BNNTs oxidized on the surface to display reactive $-\mathrm{OH}$ moieties could serve as anchors for the conjugation of monofunctional amphiphilic diblocks, leading to hybrid core-shell amphiphiles with superior physical stability and encapsulation features than regular polymeric micelles (Figure 1A). We set up to confirm the valididy of this strategy using a monofunctional PEG- $b$-PCL diblock copolymer as amphiphilic coating. The successful outcome of this study, that to the best of our knowledge represents the report on the surface modification of BNNTs by conjugation of an amphiphilic copolymer, is reported here. The PEG- $b$-PCL copolymer molecular weight of $7800 \mathrm{~g} / \mathrm{mol}$ (calculated by ${ }^{1} \mathrm{H}$ NMR) and hydrophilic-lipophilic balance of $\cong 12.8$ was synthesized by ringopening polymerization of $\varepsilon$-caprolactone (CL), using the terminal $-\mathrm{OH}$ group of a capped PEG (PEG, molecular weight of $5000 \mathrm{~g} / \mathrm{mol}$ ) as initiator in the presence of tin(II) 2-ethylhexanoate (SnOct) as catalyst (Figure 1B) ${ }^{22}$ The $M_{n}$ of the PCL block and of PEG- $b$-PCL was determined by ${ }^{1} \mathrm{H}$ NMR (in chloroform- $d$ ) using the areas of the signal at $3.3 \mathrm{ppm}$, due to the terminal methyl protons of PEG, and at $2.25 \mathrm{ppm}$ attributed to the methylene protons of PCL (Figure S1). Gel permeation chromatography (GPC) of the copolymer presented a single monomodal elution band of low polydispersity $(~ Đ=1.14)$ (Figure S2, Table S1). The diblock was reacted with maleic anhydride (MA) to form a terminal carboxylic acid group (Figure 1B). The successful modification was confirmed by ${ }^{1} \mathrm{H}$ NMR spectroscopy (Figure S3). 


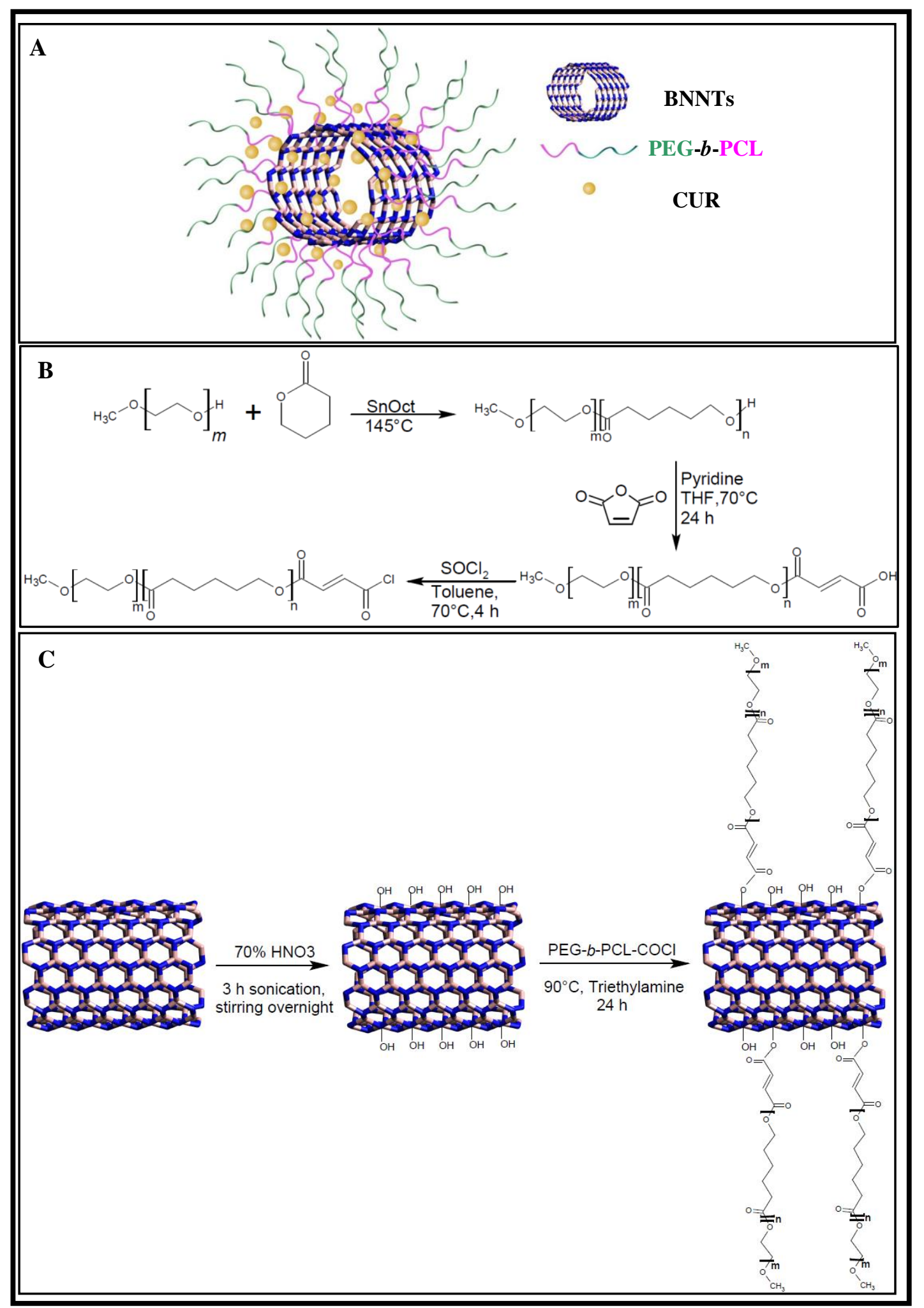

Figure 1. Synthesis of BNNT-based amphiphilic core-shell nanomaterials. (A) Schematic representation of BNNs / amphiphilic diblock copolymer conjugates. (B) Synthesis of PEG- $b$-PCL by ring-opening polymerization of $\varepsilon$-caprolactone (CL) with monomethoxy-poly(ethylene glycol) (PEG) and subsequent reactions with maleic anhydride and thionyl chloride yielding the desired acyl chloride-modified derivatives and $(\mathrm{C})$ conjugation of the reactive diblock copolymer to the oxidized surface of BNNTs 
Subsequently, the carboxylic acid was converted into the reactive acyl chloride with thionyl chloride $\left(\mathrm{SOCl}_{2}\right)$ in toluene (Figure 1B) that was conjugated to the -OH groups generated on the surface of BNNTs by oxidation with concentrated nitric acid immediately before conjugation (Figure 1C). The recoved BNNT-PCL- $b$-PEGs were thoroughly washed to remove any physically bound copolymer by dispersing in acetone, centrifuging and removing unconjugated polymer in the supernatant. This procedure was repeated several times. GPC analysis of all the PEG- $b$-PCL diblock intermediate products confirmed its chemical stability during the modification stages (Table S1). Thermogravimetric analysis (TGA) of glycine functionalized BNNTs after the cutting process ${ }^{16}$ and before treatment nitric acid showed a weight loss of $6 \% \mathrm{w} / \mathrm{W}$ at $370^{\circ} \mathrm{C}$ (Figure $\mathbf{2 A}$ ), most probably related to the degradation of glycine residues. ${ }^{23}$

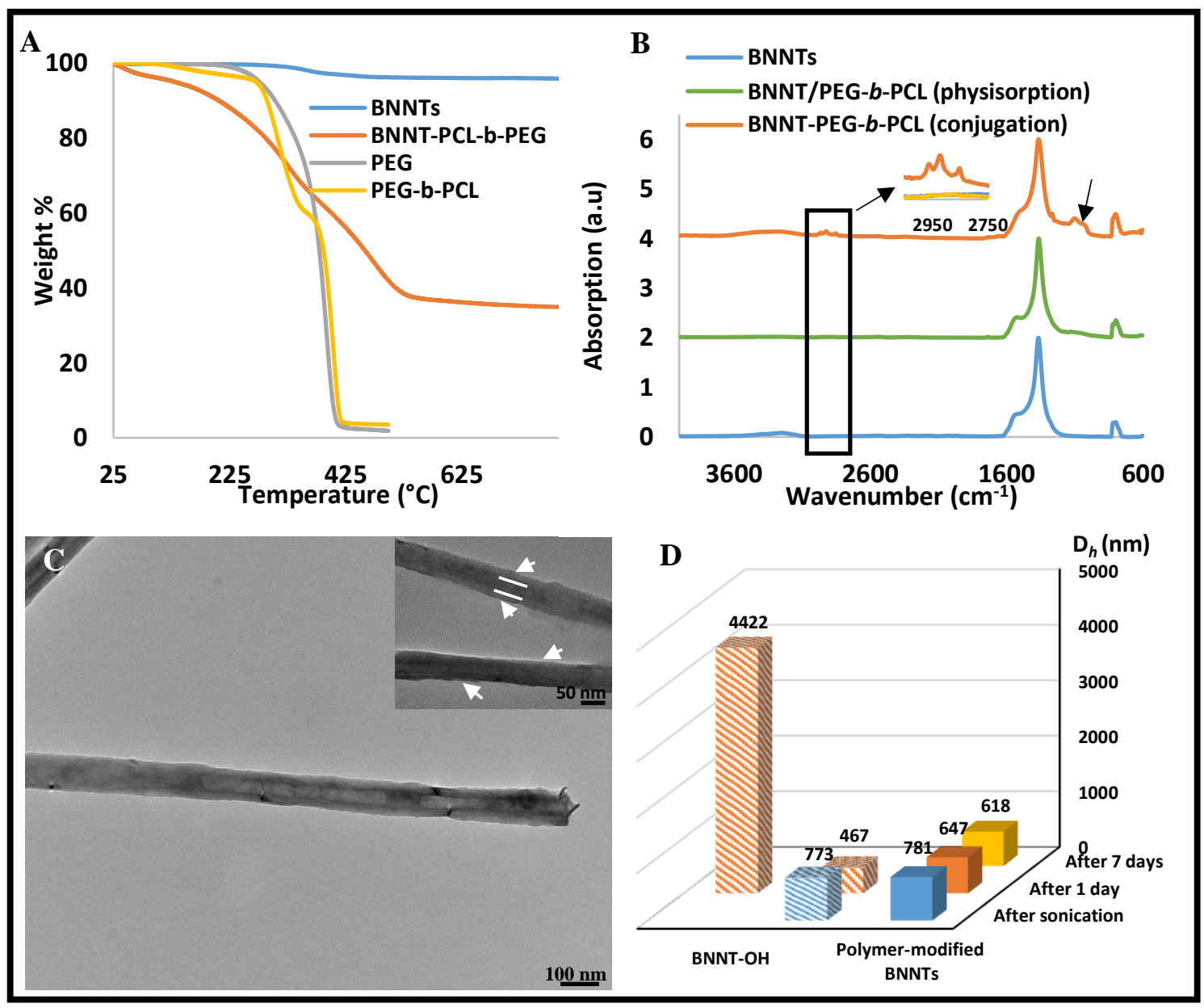

Figure 2. Characterization of the BNNT-PCL- $b$-PEG hybrid core-shell materials. $(\mathrm{A}, \mathrm{B})$ Thermograms of cut BNNTs prior to chemical modification and after PCL- $b$ PEG conjugation (BNNT-PCL- $b$-PEG), PEG- $b$-PCL and PEG; (B) FTIR of BNNTs, chemically-modified BNNT-PCL- $b$-PEG and the physical adsorption of PEG- $b$-PCL on BNNTs surface. (C) TEM image of BNNT-PCL- $b$-PEG. Insets show details of BNNTs at higher magnification. Arrows in $\mathrm{C}$ point out the thick polymeric layer 
conjugated to the surface of the nanotubes, while the lines delimit the boundaries of the inner channel. (D) Size distribution $\left(\mathrm{D}_{h}\right)$ expressed in nm of BNNT-OH (patterned bars) and BNNT-PCL- $b$-PEG (filled bars) incubated over one week, as measured by DLS.

TGA analysis of hybrid BNNT-PCL- $b$-PEG showed a weight loss of $64 \%$ w/w over a wide temperature range $\left(\sim 200-585^{\circ} \mathrm{C}\right)$ corresponding to the degradation of both PEG and PCL (Figures 2A,S4). This high conjugation extent (based on wt\%) can be probably attributed to the high surface density of - $\mathrm{OH}$ groups generated on the surface of the nanotubes upon oxidation and the very low tap density of BNNTs of approximately $0.25 \mathrm{~g} / \mathrm{cm}^{3}{ }^{24}$ The morphology and the nanostructure of the BNNTs before and after conjugation of PEG- $b$-PCL were visualized by transmission electron microscopy (TEM). Unmodified BNNTs present a smooth outer surface. The inner channel along the longitudinal axis is clearly observed (Figure S5A). PEG- $b$-PCLmodified BNNTs present a thick homogeneous outer layer that is consistent with the efficient surface conjugation (Figure 2C). The inner channel is apparent, inspite of the polymeric shell. It is worth stressing that the oxidation stage did not alter the structure of the nanotubes. TEM analysis was also used to measure the average length of the nanotubes after the cutting process and the nitric acid treatment. The average length was $2.06 \pm 0.6 \mu \mathrm{m}(\mathrm{n}=188)$ (Figure S5B). After the conjugation of the copolymer, the length did not change (data not shown). By DLS, the length of pristine BNNTs was $3.56 \pm 0.10 \mu \mathrm{m}\left(\mathrm{D}_{h}\right.$ of $\left.773 \pm 86 \mathrm{~nm}\right)$, while the average length of BNNT-PCL- $b$-PEG was $3.59 \pm 0.07 \mu \mathrm{m}\left(\mathrm{D}_{h}\right.$ of $\left.781 \pm 27 \mathrm{~nm}\right)$, confirming that the conjugation of the copolymer did not alter the size of the BNNTs. The procedure for converting $\mathrm{D}_{h}$ (that it is not the actual diameter of nanotube but the average obtained by DLS) to length can be used in case the nanotubes are well dispersed and it is presented as supporting information.

The dispersion stability of the BNNTs before and after the modification was monitored by DLS over one week, at $25^{\circ} \mathrm{C}$. At day 0 , BNNT-OH showed $\mathrm{D}_{h}$ of $773 \pm 86 \mathrm{~nm}$ (represents a length of $3.50 \mu \mathrm{m}$ ) (Figure 2D) and after one day, a bimodal size pattern with a major population with $\mathrm{D}_{h}$ of $4.4 \pm 0.4 \mu \mathrm{m}$ (5.7-fold increase in $\left.D_{h}\right)$ and a minor one of $467 \pm 237 \mathrm{~nm}$ (represents a length of $1.83 \mu \mathrm{m}$ ). These results indicated that, regardless of the presence of hydrophilic - $\mathrm{OH}$ moieties on the surface and the non-ionic surfactant Pluronic ${ }^{\circledR}$ F127 (a linear poly(ethylene oxide)- $b$-poly(propylene oxide)- $b$ poly(ethyelene oxide) copolymer) used to disperse them, most of the BNNTs 
aggregated even after 1 day; the surfactant modifies the nanotube surface by adsorption through the central hydrophobic poly(propylene oxide) block. The minor population of smaller size would suggest the presence of shorter nanotubes that were more successfully dispersed by the surfactant. However, at day 7, the instrument was not capable of measuring the $\mathrm{D}_{h}$ of $\mathrm{BNNT}-\mathrm{OH}$ due to $\mathrm{D}_{h}$ values $>10 \mu \mathrm{m}$, being in agreement with a massive aggregation. Remarkably, BNNT-PCL- $b$-PEG were very stable for at least one week owing to the steric stabilizing effect of the outer PEG layer of the conjugated copolymer (Figure 2D). The statistically significant decrease in $D_{h}$ between days 0 and $1(\mathrm{P}<0.01)$ indicated a time-dependent homogenization effect of the hydrophilic PEG shell due to a more complete dispersion of the nanotubes. ${ }^{25}$ Then, the size change was not statistically significant.

The use of inorganic nanotubes by parenteral routes (e.g., intravenous) has been proposed, though they rise serious safety concerns. ${ }^{26-28}$ Some researchers observed that oral administration of carbon nanotubes, even at very high doses $(1,000 \mathrm{mg} / \mathrm{kg}$ bodyweight), do not show toxicity. ${ }^{26}$ Moreover, nanotubes could be entrapped by mucus and thus perform as mucoadhesive nanocarriers. ${ }^{29}$ Thus, in view of their shape, the good chemical stability of PCL copolymers in water that undergo degradation at very slow rate and the envisioned good stability of the ester link between the nanotube and the diblock copolymer that is protected from the outer medium (e.g., acid gastric fluids) by the PCL coating, the hybrid core-shell materials described here were designed for ultimate use as a valuable delivery platform for non-parenteral in general and oral drug delivery in particular. Investigating the stability of the hybrid nanomaterials in gastrointestinal-like medium was beyond the scope of this work and will be studied in the future. Eventually, to prevent any hydrolysis due to the acid gastric medium and ensure full chemical stability, the nanoparticles could be encapsulated within a gastro-resistant capsule that disintegrates exclusively in the small intestine and thus, releases the cargo. In this context, next, we evaluated the cell compatibility of BNNT-OH and BNNT-PCL- $b$-PEG towards the Caco2 cell line, a standard model of the intestinal epithelium, for the first time. ${ }^{30,31}$ For this, we used the MTT [3-(4,5dimethylthiazol-2-yl)-2,5-diphenyltetrazolium bromide] assay. The time for the assay ( $24 \mathrm{~h}$ ) was chosen considering that in oral administration, the transit time is up to $6 \mathrm{~h} .{ }^{32}$ Good cell compatibility was observed at relatively low BNNT-OH and BNNT-PCL- $b$ PEG concentration $(5-10 \mu \mathrm{g} / \mathrm{mL})$. Increasing the BNNT concentration resulted in a 
slight (not statistically significant) decrease in cell viability (64-66\% ) for $50 \mu \mathrm{g} / \mathrm{mL}$ (Figure 3). Previous works reported on the good cell compatibility of BNNTs up to a concentration of $20 \mu \mathrm{g} / \mathrm{mL} .{ }^{33}$ In this sense, our findings are promising, especially considering the higher sensitivity of in vitro models with respect to in vivo ones. ${ }^{34}$ As the BNNT concentration reached $70 \mu \mathrm{g} / \mathrm{mL}$, the cytotoxicity of BNNT-OH increased significantly (viability $<40 \%$ ). In contrast, the cell viability of BNNT-PCL- $b$-PEG remained similar $(\sim 65 \%)$. This result is important as it demonstrates the outstanding contribution of the polymer shell in enhancing the cell compatibility of BNNTs. Assays utilizing more complex in vitro models of intestinal mucosa, such as comprising the coculture of Caco2 cells and mucin-secreting goblet cells, will be performed to ensure the effectiveness of the copolymer shell in increasing the compatibility of hybrid materials containing BNNTs and also assess mucoadhesion.

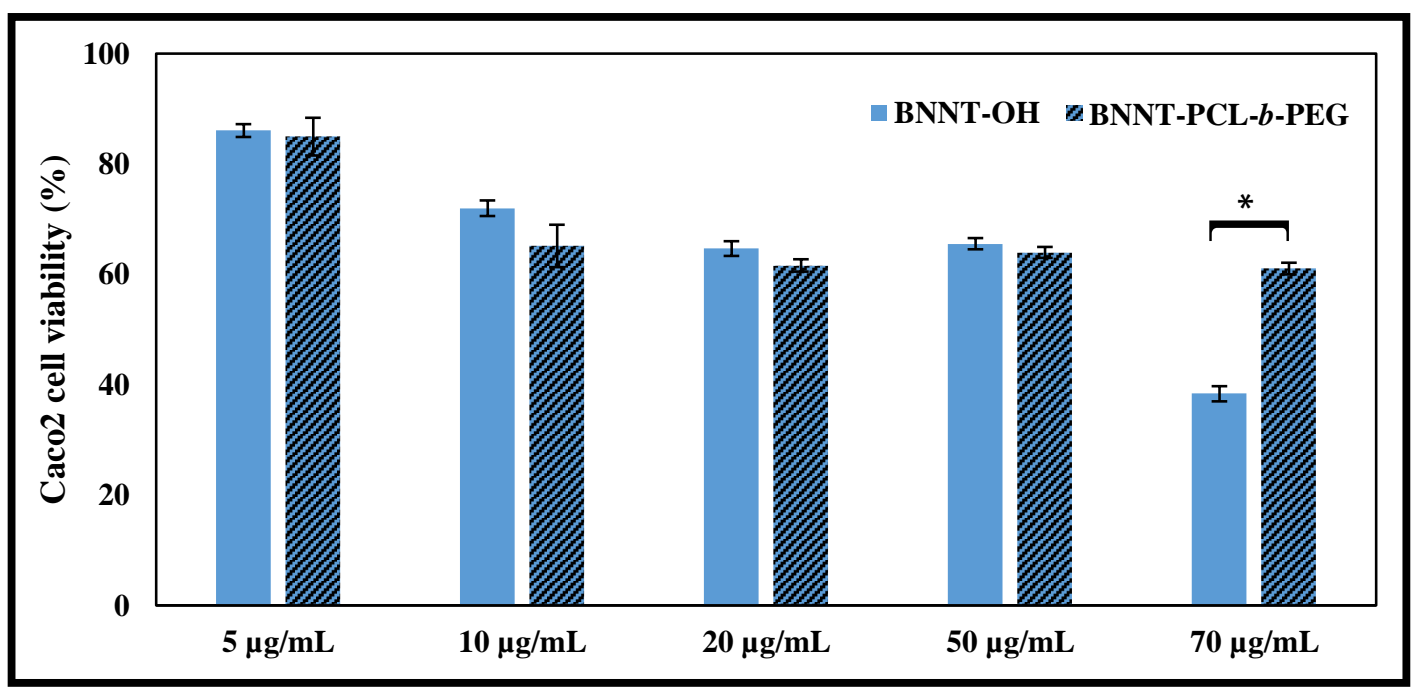

Figure 3. Caco2 cell viability. Cells were exposed to unmodified and modified BNNTs for $24 \mathrm{~h}$ and the viability estimated by MTT. *P $<0.001$ was considered statistically significant.

To challenge the encapsulation performance of the BNNT-PCL- $b$-PEG materials described here, we used curcumin (CUR), a hydrophobic molecule of very low solubility in water $(1 \mu \mathrm{g} / \mathrm{mL})$. Curcumin is a natural polyphenol investigated as antioxidant, anti-inflammatory, ${ }^{35}$ and currently in Phase II clinical trials for the chemotherapy of prostate cancer. ${ }^{36}$ However, low aqueous solubility, fast degradation, and rapid clearance all together, severely limit the oral bioavailability of CUR, jeopardizing the development of pharmaceutical products, further clinical evaluations, and its eventual approval as drug. ${ }^{37-39}$ 
The underlining assumption in designing BNNT-PCL- $b$-PEG hybrid materials was that the the hydrophobic PCL domains would act as reservoir for hydrophobic drugs, wich would strongly enhance drug loading efficiency. To test this hypothesis, we compared the CUR loading efficiency of BNNT-PCL- $b$-PEGs with the loading efficiency of glycine-treated BNNTs, ${ }^{16}$ where CUR was loaded in the BNNTs inner channel under reduced pressure.

Specifically, in a first step, we incorporated CUR inside the inner tube of BNNT-PCL$b$-PEGs by using the capillary forces as described previously. The recovered material was washed profusely with acetone to remove excess CUR and dried . In a second step, CUR was incorporated into the PCL domains by the solvent evaporation method: the recovered powder was dispersed in water; then, a CUR solution in acetone was added dropwise into the BNNTs aqueous dispersion. After acetone evaporation, the dispersion was subjected to centrifugation; the pellet was washed several times with water and then freeze-dried. ${ }^{40}$ The recovered dry powder was re-dispersed in ethanol and subjected to centrifugation. the the CUR concentration in the supernatant measured by UV spectrophotometry $(\lambda=425 \mathrm{~nm})$ employing a calibration curve in ethanol (see experimental section). The CUR load in the solid was $31.3 \% \mathrm{w} / \mathrm{w}$ (based on dry weight), representing a 313-fold increase in its apparent nanomaterial separated by centrifugation and solubility (in drug-loaded nanotubes dispersion of $1 \mathrm{mg} / \mathrm{ml}$ ) with respect to the aqueous intrinsic solubility. These findings confirm the key role played by the PCL domain in hosting the hydrophobic cargo by allowing a 2.4-fold increase of the encapsulation capacity with respect to the glycine-coated BNNTs. ${ }^{16}$ This performance is unprecedented for the encapsulation of hydrophobic molecules by BNNTs and represents an important improvement, compared to standard polymeric micelles. Next, we will investigate other boron nitride derivatives with different microstructure and morphology and their interactions with relevant biological systems.

Acknowledgements. DAS and AS thank the financial support of Technion (internal grant \#2019300), the European Union's - Seventh Framework Programme under grant agreement \#612765-MC-NANOTAR and the Russell Berrie Nanotechnology Institute (RBNI, Technion). DG is grateful to the Australian Research Council for granting a Laureate fellowship. 


\section{References}

1. X. Zhao, Z. Poon, A. C. Engler, D. K. Bonner and P. T. Hammond, Biomacromolecules, 2012, 13, 1315-1322.

2. K. M. Huh, S. C. Lee, Y. W. Cho, J. Lee, J. H. Jeong and K. Park, J. Control. Release, 2005, 101, 59-68.

3. C. Oerlemans, W. Bult, M. Bos, G. Storm, J. F. W. Nijsen and W. E. Hennink, Pharm. Res., 2010, 27, 2569-2589.

4. A. Sosnik, In: Smart Materials for Drug Delivery, C. Alvarez-Lorenzo and A. Concheiro (Eds.), Royal Society of Chemistry, pp. 115-147, 2013.

5. M. Talelli, M. Barz, C. J. F. Rijcken, F. Kiessling, W. E. Hennink and T. Lammers, Nano Today, 2015, 10, 93-117.

6. D. Abu Saleh, O. Shimoni and A. Sosnik, Mater. Today Chem., 2017, 3, 15-26.

7. R. K. O'Reilly, C. J. Hawker and K. L. Wooley, Chem. Soc. Rev., 2006, 35, $1068-1083$.

8. Y. Huang, J. Lin, J. Zou, M.-S. Wang, K. Faerstein, C. Tang, Y. Bando and D. Golberg, Nanoscale, 2013, 5, 4840-4846.

9. X. Chen, P. Wu, M. Rousseas, D. Okawa, Z. Gartner, A. Zettl and C. R. Bertozzi, J. Am. Chem. Soc., 2009, 131, 890-891.

10. J. S. M. Nithya and A. Pandurangan, RSC Adv., 2014, 4, 32031-32046.

11. F. Bouville and S. Deville, J. Am. Ceram. Soc., 2014, 97, 394-398.

12. G. Ciofani, L. Ricotti, S. Danti, S. Moscato, C. Nesti, D. D’Alessandro, D. Dinucci, F. Chiellini, A. Pietrabissa, M. Petrini and A. Menciassi, Int. J. Nanomedicine, 2010, 5, 285-298.

13. X. Wu, W. An and X. C. Zeng, J. Am. Chem. Soc., 2006, 128, 12001-12006.

14. T. Ikuno, T. Sainsbury, D. Okawa, J. M. J. Fréchet and A. Zettl, Solid State Commun., 2007, 142, 643-646.

15. Y. T. R. Lau, M. Yamaguchi, X. Li, Y. Bando, D. Golberg and F. M. Winnik, J. Phys. Chem. C, 2013, 117, 19568-19576.

16. J. Niskanen, I. Zhang, Y. Xue, D. Golberg, D. Maysinger and F. M. Winnik, Nanomedicine (Lond)., 2016, 11, 447-463.

17. G. Ciofani, G. G. Genchi, I. Liakos, A. Athanassiou, D. Dinucci, F. Chiellini and V. Mattoli, J. Colloid Interface Sci., 2012, 374, 308-314.

18. Q. Weng, B. Wang, X. Wang, N. Hanagata, X. Li, D. Liu, X. Wang, X. Jiang, Y. Bando and D. Golberg, 2014, 8, 6123-6130.

19. D. Sen Karaman, D. Desai, R. Senthilkumar, E. M. Johansson, N. Råtts, M. Odén, J. E. Eriksson, C. Sahlgren, D. M. Toivola and J. M. Rosenholm, Nanoscale Res. Lett., 2012, 7, 358.

20. G. Sharma, D. T. Valenta, Y. Altman, S. Harvey, H. Xie, S. Mitragotri and J. W. Smith, J. Control. Release, 2010, 147, 408-412.

21. V. K. Thakur, J. Yan, M.-F. Lin, C. Zhi, D. Golberg, Y. Bando, R. Sim and P. S. 
Lee, Polym. Chem., 2012, 3, 962-969.

22. A. Sosnik and D. Cohn, Polymer, 2003, 44, 7033-7042.

23. Y.-H. Yu, C.-C. Chan, Y.-C. Lai, Y.-Y. Lin, Y.-C. Huang, W.-F. Chi, C.-W. Kuo, H.-M. Lin and P.-C. Chen, RSC Adv., 2014, 4, 56373-56384.

24. G. G. Genchi, A. Rocca, A. Grillone, A. Marino and G. Ciofanni, In: Boron Nitride Nanotubes in Nanomedicine, G. Ciofani and V. Mattoli (Eds.), Elsevier, pp. 201-214, 2016.

25. H. Wang, Thermal Conductivity 27: Thermal Expansion 15, 2005.

26. W. Zhang, Z. Zhang and Y. Zhang, Nanoscale Res. Lett., 2011, 6, Art. 555.

27. B. S. Wong, S. L. Yoong, A. Jagusiak, T. Panczyk, H. K. Ho, W. H. Ang and G. Pastorin, Adv. Drug Deliv. Rev., 2013, 65, 1964-2015.

28. Y.-Y. Xu, J. Ge, M.-H. Zhang, W.-J. Sun, J. Zhang, P.-L. Yu, Y.-F. Zheng, J. Yang and X.-Q. Zhu, Int. J. Toxicol., 2016, 35, 634-643.

29. J. K. de Kruif, G. Ledergerber, C. Garofalo, E. Fasler-Kan, M. Kuentz, Eur. J. Pharm. Biopharm., 2016, 101, 90-102.

30. Y. Sambuy, I. De Angelis, G. Ranaldi, M. L. Scarino, A. Stammati and F. Zucco, Cell Biol. Toxicol., 2005, 21, 1-26.

31. V. Meunier, M. Bourrie, Y. Berger and G. Fabre, Cell Biol. Toxicol., 1995, 11, 187-194.

32. Y. Ma, A. V. Fuchs, N. R. B. Boase, B. E. Rolfe, A. G. A. Coombes and K. J. Thurecht, Eur. J. Pharm. Biopharm., 2015, 94, 393-403.

33. G. Ciofani, S. Del Turco, A. Rocca, G. de Vito, V. Cappello, M. Yamaguchi, X. Li, B. Mazzolai, G. Basta, M. Gemmi, V. Piazza, D. Golberg and V. Mattoli. Nanomedicine (Lond.), 2014, 9, 773-788.

34. H. H. Gustafson, D. Holt-Casper, D. W. Grainer, H. Ghandehari, Nano Today, 2015, 10, 487-510.

35. R. Motterlini, R. Foresti, R. Bassi and C. J. Green, Free Radic. Biol. Med., 2000, 28, 1303-1312.

36. Y. Lotan, Curcumin in Treating Patients with Prostate Cancer That Was Removed by Surgery, https://www.cancer.gov/about-cancer/treatment/clinicaltrials/search/view?cdrid $=758229 \&$ version $=$ HealthProfessional\&protocolsearch $\mathrm{id}=6892770$.

37. C. Mohanty and S. K. Sahoo, Biomaterials, 2010, 31, 6597-6611.

38. S. C. Gupta, S. Patchva and B. B. Aggarwal, AAPS J., 2013, 15, 195-218.

39. P. Anand, A. B. Kunnumakkara, R. A. Newman, B. B. Aggarwal, P. Anand, A. B. Kunnumakkara and R. A. Newman, 2007, 4, 807-818.

40. A. Anitha, V. G. Deepagan, V. V. Divya Rani, D. Menon, S. V. Nair and R. Jayakumar, Carbohydr. Polym., 2011, 84, 1158-1164. 